\title{
Performance of electron, photon and muon triggers at the CMS High Level Trigger
}

Thiago R. F. P. Tomei*

On behalf of the CMS Collaboration

Instituto de Física Teórica, Universidade Estadual Paulista

E-mail: Thiago.Tomeidcern.ch

The trigger systems of the LHC detectors play a crucial role in determining the physics capabilities of the experiments. A reduction of several orders of magnitude of the event rate is needed to reach values compatible with the detector readout, offline storage and analysis capabilities. The CMS experiment has been designed with a two-level trigger system: the Level 1 (L1) Trigger, implemented on custom-designed electronics, and the High Level Trigger (HLT), a streamlined version of the CMS reconstruction and analysis software running on a computer farm. Here we will present the design and performance of the main muon, electron and photon triggers, in view of the more challenging conditions for the LHC Run 2. For the muon case, we discuss the improvements in the isolation algorithm with the usage of Particle Flow techniques, which allow for better discrimination power between processes with prompt muons and the the effect of jets penetrating through the hadronic calorimeter into the muon chambers. For the electron and photon cases, we discuss the identification variables used to distinguish between prompt electrons/photons and hadronic jets enriched in electromagnetic component.

XXVII International Symposium on Lepton Photon Interactions at High Energies 17-22 August 2015

Ljubljana, Slovenia

${ }^{*}$ Speaker. 


\section{Introduction}

With the beginning of the LHC Run 2, the CMS experiment has to cope with harsher operating conditions: higher centre-of-mass energy of $13 \mathrm{TeV}$, leading to an enhancement of the production cross section of interesting processes like weak boson and top quark production, and larger occurrence of multiple proton-proton interactions in the same / near bunch crossings (pileup), ranging from 20 to 40 simultaneous interactions. The High-Level Trigger software [1] has been optimised for these conditions, balancing a high selection efficiency on signal events while keeping the output rate at acceptable levels both for the online data acquisition system and the offline prompt reconstruction center. In this paper we discuss the improvements made for $e, \gamma$ and $\mu$ trigger algorithms.

\section{Muon Triggers}

Muon candidates coming from weak boson processes have the characteristic of being isolated from the rest of the activity in the event. A new approach to isolation has been developed for Run 2, based on Particle Flow clusters (exploring the whole detector granularity) and sequential isolation (having separate isolation definitions for ECAL, HCAL and the tracker). Those improvements allow to maximise the selection efficiency for isolated muons while keeping similar kinematic thresholds as those used at the end of Run 1. Figure 1 shows the comparison of the isolation algorithm performance for the 2012 and 2015 configurations.

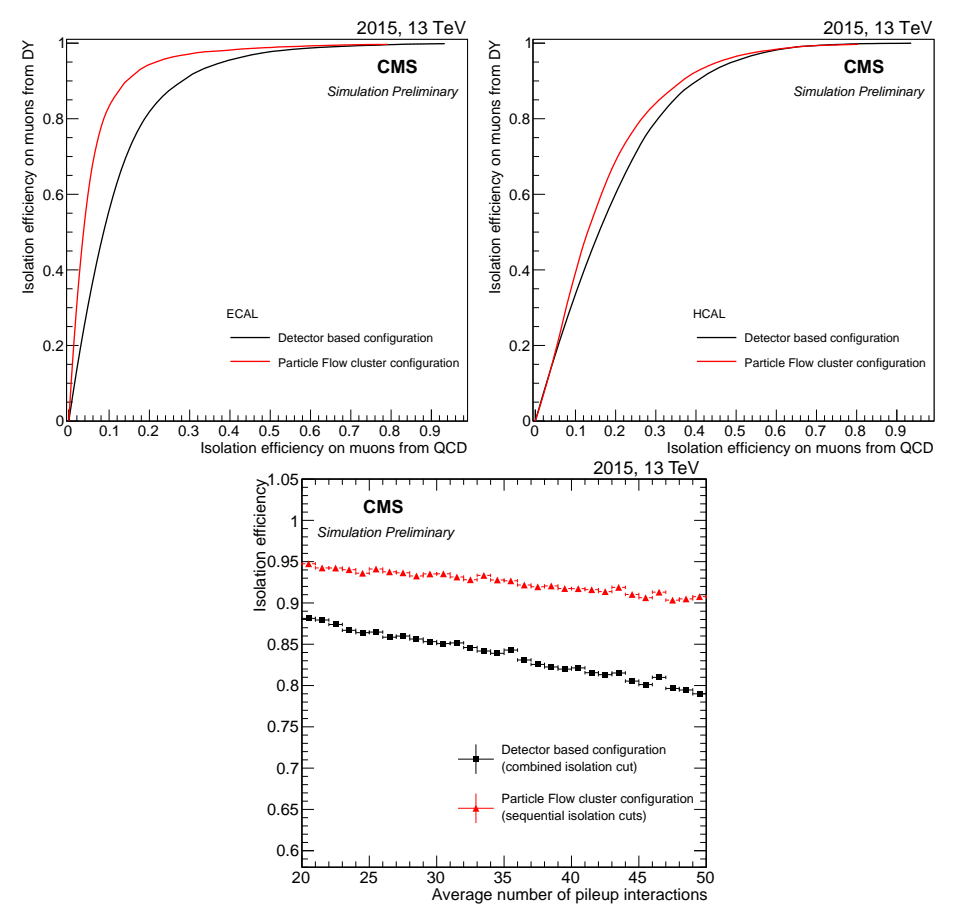

Figure 1: Top left (top right) panel: performance of the ECAL (HCAL) calorimeter isolation, for $\mathrm{Z} \rightarrow \mu \mu$ signal events vs. multijet background events, for an average pileup of 40 and bunch spacing of $25 \mathrm{~ns}$. Bottompanel: isolation efficiency as function of the average number of pileup interactions for two working points giving a similar rate reduction. The comparison is between the detector based setup (black) and the Particle Flow (red) algorithm used in the 2015 configuration. 


\section{Electron and Photon Triggers}

The identification of electrons and photons at CMS relies on the granularity of ECAL and on the high performance of the tracker, both during off-line reconstruction and at the HLT. The main handles to separate prompt electrons and photons from jets particularly rich in electromagnetic (EM) component are:

- the energy weighted pseudorapidity-width $\sigma_{\eta}$ of the EM cluster;

- the hadronic energy deposit behind the EM cluster, and the ratio $H / E$ of those energies.

- the difference between the inverses of the EM cluster energy and of the associated electron track momentum $(1 / E-1 / p)$.

- the absolute values of pseudorapidity and azimuthal angle of the difference between the electron's cluster and track $(\Delta \eta$ and $\Delta \phi)$.

Figure 2 shows the distribution of those variables for simulated Drell-Yan and multijet events, with representative thresholds.
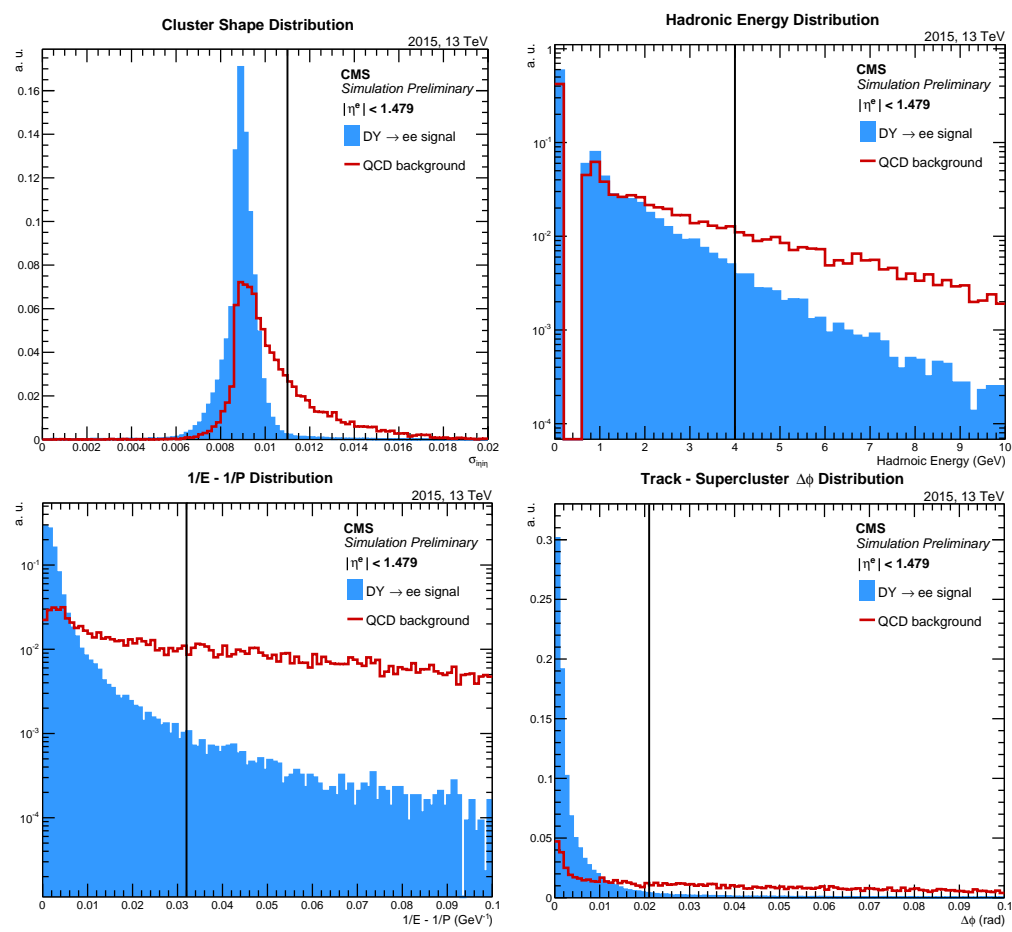

Figure 2: Distribution of the identification variables for events from Drell-Yan processes (blue full histograms) and for multijet background processes (red line histograms). Further identification techniques are available for the design of triggers, such as matching between the EM cluster and the pixel seed, number of expected missing hits in active tracker layers and lepton isolation.

For the electron case, CMS employed isolation techniques to allow for lower kinematic thresholds on the trigger algorithms. The isolation used in the electron triggers is akin to that used in the muon triggers, employing both Particle Flow clusters and reconstructed tracks. The usage of isolation allows the $\mathrm{p}_{\mathrm{T}}$ threshold of the single electron trigger to go as low as $23 \mathrm{GeV}$, ensuring its usability for the measurement of electroweak, top and Higgs processes. 


\section{Displaced Leptons}

Displaced leptons can arise from some Beyond Standard Model processes where a heavy, long-lived neutral particle decays far away from the luminous region. Generally, photon triggers are reasonably efficient for displaced electrons, while for muons special provisions must be in place to ensure proper $\mathrm{p}_{\mathrm{T}}$ estimation. Figure 3 shows the fraction of simulated signal events passing the special-purpose trigger, as a function of the HLT $\mathrm{p}_{\mathrm{T}}$ requirement for the trigger configurations used in 2012 and 2015. The trigger algorithm selects displaced and out-of-time muons, while the 2015 configuration improves the $\mathrm{p}_{\mathrm{T}}$ resolution for such muons. The trigger rate is kept under control $-\mathscr{O}(10 \mathrm{~Hz})$, mostly depending on the LHC filling scheme - through the usage of bunch-crossing veto bits.

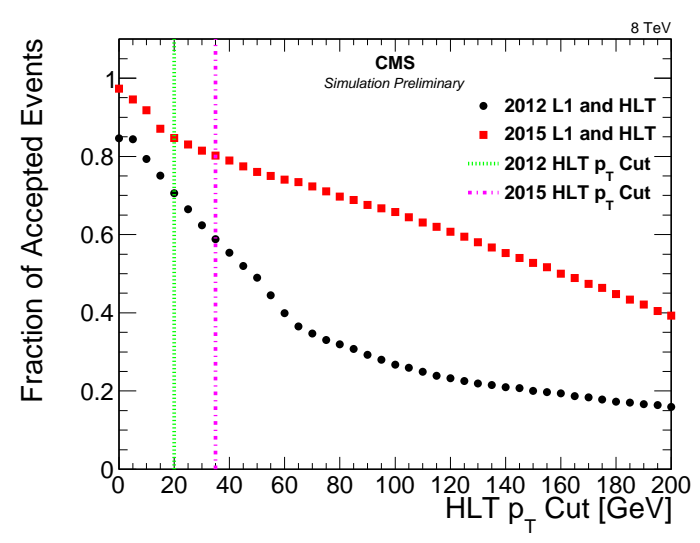

Figure 3: Fraction of simulated signal events passing the special-purpose trigger with respect to the generator level, as a function of the HLT $\mathrm{p}_{\mathrm{T}}$ requirement, for the trigger configuration in 2012 (black) and the configuration to be used in 2015 (red). The simulated signal is the production of long-lived leptons ( $q=2 \mathrm{e}$, $\mathrm{m}=500 \mathrm{GeV}$ ) stopping in the detector (typically a few meters from the interaction point) and decaying to 2 back-to-back muons.

\section{Conclusion}

The updated configuration of the High Level Trigger was successfully deployed during the 2015 run, allowing CMS to record data equivalent to approximately $3.6 \mathrm{fb}^{-1}$ of proton-proton collisions at $13 \mathrm{TeV}$ [2]. As a result of the improvements to the HLT software, we were able to keep the kinematic object thresholds close to those used during Run 1. For the muon case, the lowest single isolated trigger used in $2015 \mathrm{had}$ a $\mathrm{p}_{\mathrm{T}}$ threshold of $18 \mathrm{GeV}$; for the electron and photon cases, the lowest single isolated trigger had a $\mathrm{p}_{\mathrm{T}}$ threshold of $23 \mathrm{GeV}$ (electrons) and $165 \mathrm{GeV}$ (photons). The CMS collaboration now proceeds to the analysis of this dataset.

\section{References}

[1] P. Sphicas [CMS Collaboration], "CMS: The TriDAS project. Technical Design Report, Vol. 2: Data Acquisition and High-Level Trigger", CERN-LHCC-2002-026.

[2] CMS Collaboration, "CMS Luminosity Public Results", https://twiki.cern.ch/twiki/bin/view/CMSPublic/LumiPublicResults. 